\title{
Travelling Wave Solutions for the Long-Short-Wave Interaction System Using the Unified Method
}

\author{
Şamil AKÇAĞIL* \\ Bilecik Şeyh Edebali University, Vocational School of Pazaryeri, Bilecik
}

\begin{abstract}
The Long-Short-Wave interaction system plays a significant role between low frequency long waves and high frequency short waves. Besides being an important complex model it is also related with several physical phenomenon such as, gravity and water waves, plasma and bio-physics, nonlinear optic. This system was handled by many researchers and solutions of this system were obtained by using different methods. In this paper, new travelling wave solutions for the Long-short-wave interaction system are formaly drived by using different method namely the unified method. The obtained solutions can be considered as improved version of the previous solutions. This study also shows the efficiency of the unified method. Mathematica and Maple facilitates the tedious algebraic calculations.
\end{abstract}

Keywords: Travelling Wave Solution, Long-Short-Wave Interaction System, The Unified Method.

\section{Unified Yöntem Kullanılarak Uzun-Kısa-Dalga Etkileşimi Sisteminin Hareketli Dalga Çözümlerinin Elde Edilmesi}

\begin{abstract}
$\ddot{\mathbf{O} z}$
Uzun Kısa Dalga etkileşimi sistemi, düşük frekanslı uzun dalgalar ve yüksek frekanslı kısa dalgalar arasında önemli bir rol oynar. Önemli bir kompleks model olmasının yanı sıra, yerçekimi ve su dalgaları, plazma ve biyofizik, doğrusal olmayan optik gibi çeşitli fiziksel olgularla da ilgilidir. Bu sistem birçok araştırmacı tarafından ele alınmış ve sistemin farklı yöntemler kullanılarak bir çok çözümü elde edilmiştir. Bu çalışmada, uzun kısa dalga etkileşim sisteminin yeni hareketli dalga çözümleri, unified yöntem adı verilen yöntemle elde edilecektir. Elde edilen çözümler önceki çözümlerin geliştirilmiş versiyonu olarak düşünülebilir. Bu çalışma aynı zamanda unified yöntemin etkinliğini de göstermektedir. Uzun cebirsel işlemler için Maple ve Mathematica kullanılacaktır.
\end{abstract}

Anahtar kelimeler: Hareketli Dalga Çözüm, Uzun-Kısa-Dalga Etkileşimi Sistemi, Unified Yöntem.

\section{Introduction}

Let $\psi(x, t)$ is a complex variable function and $v(x, t)$ is a real variable function. The long-short-wave interaction system (LSWIS) is given by

$$
\begin{gathered}
i \psi_{t}+\psi_{x x}-\psi v=0 \\
v_{t}+v_{x}+\left(\left|\psi^{2}\right|\right)_{x}=0
\end{gathered}
$$

In this system of equations, $x$ represents the spatial coordinate and $t$ represents time. The interaction between short and long waves is expressed by this system. Wang et al have considered LSWIS and obtained various periodic wave solutions expressed by Jacobi elliptic function of the system in terms of the F-expansion method [1]. The periodic and solitary wave solutions of the system were obtained using the first integral method [2]. Sirendaoerji constructed the infinite number of exact traveling wave solutions of the LSWIS via an extended tanh-function method [3].

"Sorumlu yazar: samilakcagil@hotmail.com

Geliş Tarihi: 18/04/2018 Kabul Tarihi: 05/06/2018 
Previously, conventional methods such as Laplace and Fourier transforms were used to solve partial differential equations. These transformations have made the solutions easier because they have transformed the differential equations into algebraic equations. Using these integral transformations, however, involved many complex algebraic operation. In the recent years, with the production of powerful computers, it has become easier to solve these mixed algebraic operations. As a result, it allowed a major step in the current calculation methods and led to the discovery of powerful solution methods. One of these methods was the tanh-coth method. In the last years, based on this method, many researchers have introduced another modification of this method and put it into practice. For more information, we refer the readers to [4-9] and the references therein.

In an our previous work [10], we have compared main hyperbolic methods in the literature and introduced a new method namely "the unified method" that can be used instead of all of them. We have also proved that this method gives all of the solutions obtained by all of them.

In this work, the LSWIS has been considered and obtained new solutions by using the unifid method. Even more, all of the solutions obtained using different methods have been handled in only one method.

\section{Despription of the Unified Method}

The unified method can be summarized as follows:

(i) First, consider a nonlinear partial differential equation (NPDE)

$P\left(u, u_{t}, u_{x}, u_{x t}, u_{t t}, u_{x x}, \ldots\right)=0$.

(ii) To find the travelling wave solutions of (2), use the wave variable $\xi=x-c t$ so that

$u(x, t)=U(\xi)$.

(iii) Using (3) changes PDE (2) to an ODE.

$Q\left(U, c U^{\prime}, U^{\prime}, c U^{\prime \prime}, c^{2} U^{\prime \prime}, U^{\prime \prime}, \ldots\right)=0$.

(iv) If necessary one integrates (4) as many times as possible. Adhering to the boundary conditions

$U(\xi) \rightarrow 0, \frac{d^{n} U(\xi)}{d \xi} \rightarrow 0(n=1,2,3, \ldots)$ for $\xi \rightarrow \mp \infty$

the integration constants should all be set zero.

(v) Suppose the solution of (2) can be expressed by a polynomial in $\varphi$ as follows:

$u(\xi)=a_{0}+\sum_{i=1}^{M}\left(a_{i} \varphi^{i}+b_{i} \varphi^{-i}\right)$

where $\varphi=\varphi(\xi)$ satisfies the equation

$\varphi^{\prime}(\xi)=\varphi^{2}(\xi)+\alpha$

where $\varphi^{\prime}=\frac{d \varphi}{d \xi}$ and $a_{i}, b_{i}$ and $\alpha$ are constants. $M$ is a positive integer in most cases. If $M$ is not an integer, this problem can be solved by using a transformation formula. The general solution of (6) as follows: 
Family 1. When $\alpha<0$,

$$
\varphi(\xi)=\left\{\begin{array}{c}
\frac{\sqrt{-\left(A^{2}+B^{2}\right) \alpha}-A \sqrt{-b} \cosh \left(2 \sqrt{-\alpha}\left(\xi+\xi_{0}\right)\right)}{A \sinh \left(2 \sqrt{-\alpha}\left(\xi+\xi_{0}\right)\right)+B} \\
\frac{-\sqrt{-\left(A^{2}+B^{2}\right) \alpha}-A \sqrt{-\alpha} \cosh \left(2 \sqrt{-\alpha}\left(\xi+\xi_{0}\right)\right)}{A \sinh \left(2 \sqrt{-\alpha}\left(\xi+\xi_{0}\right)\right)+B} \\
\sqrt{-\alpha}+\frac{-2 A \sqrt{-\alpha}}{A+\cosh \left(2 \sqrt{-\alpha}\left(\xi+\xi_{0}\right)\right)-\sinh \left(2 \sqrt{-\alpha}\left(\xi+\xi_{0}\right)\right)} \\
-\sqrt{-\alpha}+\frac{2 A \sqrt{-\alpha}}{A+\cosh \left(2 \sqrt{-\alpha}\left(\xi+\xi_{0}\right)\right)+\sinh \left(2 \sqrt{-\alpha}\left(\xi+\xi_{0}\right)\right)}
\end{array}\right.
$$

Family 2. When $\alpha>0$,

$$
\varphi(\xi)=\left\{\begin{array}{c}
\frac{\sqrt{\left(A^{2}-B^{2}\right) \alpha}-A \sqrt{\alpha} \cos \left(2 \sqrt{\alpha}\left(\xi+\xi_{0}\right)\right)}{A \sin \left(2 \sqrt{\alpha}\left(\xi+\xi_{0}\right)\right)+B} \\
\frac{-\sqrt{\left(A^{2}-B^{2}\right) \alpha}-A \sqrt{b} \cos \left(2 \sqrt{\alpha}\left(\xi+\xi_{0}\right)\right)}{A \sin \left(2 \sqrt{\alpha}\left(\xi+\xi_{0}\right)\right)+B} \\
i \sqrt{\alpha}+\frac{-2 A i \sqrt{\alpha}}{A+\cos \left(2 \sqrt{\alpha}\left(\xi+\xi_{0}\right)\right)-i \sin \left(2 \sqrt{\alpha}\left(\xi+\xi_{0}\right)\right)} \\
-i \sqrt{\alpha}+\frac{2 A i \sqrt{\alpha}}{A+\cos \left(2 \sqrt{\alpha}\left(\xi+\xi_{0}\right)\right)+i \sin \left(2 \sqrt{\alpha}\left(\xi+\xi_{0}\right)\right)}
\end{array}\right.
$$

Family 3. When $\alpha=0$,

$\varphi(\xi)=-\frac{1}{\xi+\xi_{0}}$

where $A$ and $B$ are two real arbitrary constants, $\xi_{0}$ is an arbitrary constant and $i=\sqrt{-1}$.

(vi) To determine the parameter $M$, the linear terms of highest order with the highest-order nonlinear terms in (4) are balanced.

(vii) When $M$ determined, using (5) and (6) into (4) one gets a polynomial of $\varphi$. All the coefficients of powers of $\varphi$ in the resulting equation must be zero. This yields a set of algebraic equations for $a_{i}, b_{i}, c$ and $\alpha$.

(viii) Solving the equation system and using (5), one obtains an analytic solution in a closed form of (2) depending on the value of $\alpha$.

\section{Travelling Wave Solutions of the LSWIS}

Using the following transformation to (1)

$\psi(x, t)=e^{i \theta} U(\xi), v(x, t)=V(\xi)$

$\theta=m x+n t, \xi=x-c t$

where $m$ and $n$ are real constants, $u(x, t)$ and $v(x, t)$ are real functions. If we substitute (10) into (1) then we get $c=2 m$. The equation system (1) turns into a system of ODE as follows:

$U^{\prime \prime}-\left(n+m^{2}\right) U-U V=0$

$(2 m-1) V^{\prime}-2 U U^{\prime}=0$.

If one integrates (12) and considers the constant of integration to be zero, then finds 
$V=\frac{U^{2}}{2 m-1}$

Setting (13) into (11), we get only one ODE in the following form:

$U^{\prime \prime}-\left(n+m^{2}\right) U-\frac{1}{2 m-1} U^{3}=0$.

Balancing $U^{\prime \prime}$ with $U^{3}$ in (14) gives $M=1$. Using the finite expansion

$u(\xi)=a_{0}+\sum_{i=1}^{1}\left(a_{i} \varphi^{i}+b_{i} \varphi^{-i}\right)$

the solutions of (14) must be in the form

$u(\xi)=a_{0}+a_{1} \varphi+b_{1} \varphi^{-1}$

If we substitute (15) into (14) the collect the coefficients of each power of $\varphi$ we get a system of equations. Solving this system for $a_{0}, a_{1}, b_{1}$ and $c$, we obtain following four sets of solutions:

\section{Set 1:}

$a_{0}=0, \quad a_{1}= \pm \sqrt{-2+4 m}, \quad b_{1}=0, \quad c= \pm 2 \sqrt{-n+2 \alpha}$

\section{Set 2:}

$a_{0}=0, a_{1}=0, \quad b_{1}= \pm \sqrt{-2+4 m} \alpha, \quad c= \pm 2 \sqrt{-n+2 \alpha}$

\section{Set 3:}

$a_{0}=0, \quad a_{1}= \pm \sqrt{-2+4 m} \alpha, \quad b_{1}= \pm \sqrt{-2+4 m}, \quad c= \pm 2 \sqrt{-n-4 \alpha}$

\section{Set 4:}

$a_{0}=0, \quad a_{1}= \pm \sqrt{-2+4 m}, b_{1}=\mp \sqrt{-2+4 m} \alpha, c= \pm 2 \sqrt{-n+8 \alpha}$

These four sets give the solutions respectively:

$$
\begin{aligned}
& \psi_{1}= \pm \sqrt{-2+4 m} e^{i \theta}\left(\frac{\sqrt{\left(A^{2}-B^{2}\right) \alpha}-A \sqrt{\alpha} \cos \left(2 \sqrt{\alpha}\left(\xi+\xi_{0}\right)\right)}{A \sin \left(2 \sqrt{\alpha}\left(\xi+\xi_{0}\right)\right)+B}\right) \\
& v_{1}=2 e^{2 i \theta}\left(\frac{\sqrt{\left(A^{2}-B^{2}\right) \alpha}-A \sqrt{\alpha} \cos \left(2 \sqrt{\alpha}\left(\xi+\xi_{0}\right)\right)}{A \sin \left(2 \sqrt{\alpha}\left(\xi+\xi_{0}\right)\right)+B}\right)^{2} \\
& \psi_{2}= \pm \sqrt{-2+4 m} e^{i \theta}\left(\frac{-\sqrt{\left(A^{2}-B^{2}\right) \alpha}-A \sqrt{\alpha} \cos \left(2 \sqrt{\alpha}\left(\xi+\xi_{0}\right)\right)}{A \sin \left(2 \sqrt{\alpha}\left(\xi+\xi_{0}\right)\right)+B}\right) \\
& v_{2}=2 e^{2 i \theta}\left(\frac{-\sqrt{\left(A^{2}-B^{2}\right) \alpha}-A \sqrt{\alpha} \cos \left(2 \sqrt{\alpha}\left(\xi+\xi_{0}\right)\right)}{A \sin \left(2 \sqrt{\alpha}\left(\xi+\xi_{0}\right)\right)+B}\right)^{2} \\
& \psi_{3}= \pm \sqrt{-2+4 m} e^{i \theta}\left(i \sqrt{\alpha}+\frac{-2 A i \sqrt{\alpha}}{A+\cos \left(2 \sqrt{\alpha}\left(\xi+\xi_{0}\right)\right)-i \sin \left(2 \sqrt{\alpha}\left(\xi+\xi_{0}\right)\right)}\right)
\end{aligned}
$$




$$
\begin{aligned}
& v_{3}=2 e^{2 i \theta}\left(i \sqrt{\alpha}+\frac{-2 A i \sqrt{\alpha}}{A+\cos \left(2 \sqrt{\alpha}\left(\xi+\xi_{0}\right)\right)-i \sin \left(2 \sqrt{\alpha}\left(\xi+\xi_{0}\right)\right)}\right)^{2} \\
& \psi_{4}= \pm \sqrt{-2+4 m} e^{i \theta}\left(-i \sqrt{\alpha}+\frac{2 A i \sqrt{\alpha}}{A+\cos \left(2 \sqrt{\alpha}\left(\xi+\xi_{0}\right)\right)+\sin \left(2 \sqrt{\alpha}\left(\xi+\xi_{0}\right)\right)}\right) \\
& v_{4}=2 e^{2 i \theta}\left(-i \sqrt{\alpha}+\frac{2 A i \sqrt{\alpha}}{A+\cos \left(2 \sqrt{\alpha}\left(\xi+\xi_{0}\right)\right)+\sin \left(2 \sqrt{\alpha}\left(\xi+\xi_{0}\right)\right)}\right)^{2} \\
& \psi_{5}= \pm \sqrt{-2+4 m} \alpha e^{i \theta}\left(\frac{\sqrt{\left(A^{2}-B^{2}\right) \alpha}-A \sqrt{\alpha} \cos \left(2 \sqrt{\alpha}\left(\xi+\xi_{0}\right)\right)}{A \sin \left(2 \sqrt{\alpha}\left(\xi+\xi_{0}\right)\right)+B}\right)^{-1} \\
& v_{5}=2 \alpha^{2} e^{2 i \theta}\left(\frac{\sqrt{\left(A^{2}-B^{2}\right) \alpha}-A \sqrt{\alpha} \cos \left(2 \sqrt{\alpha}\left(\xi+\xi_{0}\right)\right)}{A \sin \left(2 \sqrt{\alpha}\left(\xi+\xi_{0}\right)\right)+B}\right)^{-2}
\end{aligned}
$$$$
\psi_{6}= \pm \sqrt{-2+4 m} \alpha e^{i \theta}\left(\frac{-\sqrt{\left(A^{2}-B^{2}\right) \alpha}-A \sqrt{\alpha} \cos \left(2 \sqrt{\alpha}\left(\xi+\xi_{0}\right)\right)}{A \sin \left(2 \sqrt{\alpha}\left(\xi+\xi_{0}\right)\right)+B}\right)^{-1}
$$$$
v_{6}=2 \alpha^{2} e^{2 i \theta}\left(\frac{-\sqrt{\left(A^{2}-B^{2}\right) \alpha}-A \sqrt{\alpha} \cos \left(2 \sqrt{\alpha}\left(\xi+\xi_{0}\right)\right)}{A \sin \left(2 \sqrt{\alpha}\left(\xi+\xi_{0}\right)\right)+B}\right)^{-2}
$$$$
\psi_{7}= \pm \sqrt{-2+4 m} \alpha e^{i \theta}\left(i \sqrt{\alpha}+\frac{-2 A i \sqrt{\alpha}}{A+\cos \left(2 \sqrt{\alpha}\left(\xi+\xi_{0}\right)\right)-i \sin \left(2 \sqrt{\alpha}\left(\xi+\xi_{0}\right)\right)}\right)^{-1}
$$$$
v_{7}=2 \alpha^{2} e^{2 i \theta}\left(i \sqrt{\alpha}+\frac{-2 A i \sqrt{\alpha}}{A+\cos \left(2 \sqrt{\alpha}\left(\xi+\xi_{0}\right)\right)-i \sin \left(2 \sqrt{\alpha}\left(\xi+\xi_{0}\right)\right)}\right)^{-2}
$$

$\psi_{8}= \pm \sqrt{-2+4 m} \alpha e^{i \theta}\left(-i \sqrt{\alpha}+\frac{2 A i \sqrt{\alpha}}{A+\cos \left(2 \sqrt{\alpha}\left(\xi+\xi_{0}\right)\right)+\sin \left(2 \sqrt{\alpha}\left(\xi+\xi_{0}\right)\right)}\right)^{-1}$

$v_{8}=2 \alpha^{2} e^{2 i \theta}\left(-i \sqrt{\alpha}+\frac{2 A i \sqrt{\alpha}}{A+\cos \left(2 \sqrt{\alpha}\left(\xi+\xi_{0}\right)\right)+\sin \left(2 \sqrt{\alpha}\left(\xi+\xi_{0}\right)\right)}\right)^{-2}$

where $\xi=x-c t, c= \pm 2 \sqrt{-n+2 \alpha}, \alpha>0, A$ and $B$ are two real constants;

$$
\begin{aligned}
& \psi_{9}= \pm \sqrt{-n+4 m} e^{i \theta}\left[\left(\frac{\sqrt{\left(A^{2}-B^{2}\right) \alpha}-A \sqrt{\alpha} \cos \left(2 \sqrt{\alpha}\left(\xi+\xi_{0}\right)\right)}{A \sin \left(2 \sqrt{\alpha}\left(\xi+\xi_{0}\right)\right)+B}\right)+\alpha\left(\frac{\sqrt{\left(A^{2}-B^{2}\right) \alpha}-A \sqrt{\alpha} \cos \left(2 \sqrt{\alpha}\left(\xi+\xi_{0}\right)\right)}{A \sin \left(2 \sqrt{\alpha}\left(\xi+\xi_{0}\right)\right)+B}\right)^{-1}\right] \\
& v_{9}=\frac{-n+4 m}{2 m-1} e^{2 i \theta}\left[\left(\frac{\sqrt{\left(A^{2}-B^{2}\right) \alpha}-A \sqrt{\alpha} \cos \left(2 \sqrt{\alpha}\left(\xi+\xi_{0}\right)\right)}{A \sin \left(2 \sqrt{\alpha}\left(\xi+\xi_{0}\right)\right)+B}\right)+\alpha\left(\frac{\sqrt{\left(A^{2}-B^{2}\right) \alpha}-A \sqrt{\alpha} \cos \left(2 \sqrt{\alpha}\left(\xi+\xi_{0}\right)\right)}{A \sin \left(2 \sqrt{\alpha}\left(\xi+\xi_{0}\right)\right)+B}\right)^{-1}\right]^{2} \\
& \psi_{10}= \pm \sqrt{-n+4 m} e^{i \theta}\left[\left(\frac{-\sqrt{\left(A^{2}-B^{2}\right) \alpha}-A \sqrt{\alpha} \cos \left(2 \sqrt{\alpha}\left(\xi+\xi_{0}\right)\right)}{A \sin \left(2 \sqrt{\alpha}\left(\xi+\xi_{0}\right)\right)+B}\right)+\alpha\left(\frac{-\sqrt{\left(A^{2}-B^{2}\right) \alpha}-A \sqrt{\alpha} \cos \left(2 \sqrt{\alpha}\left(\xi+\xi_{0}\right)\right)}{A \sin \left(2 \sqrt{\alpha}\left(\xi+\xi_{0}\right)\right)+B}\right)^{-1}\right] \\
& v_{10}=\frac{-n+4 m}{2 m-1} e^{2 i \theta}\left[\left(\frac{-\sqrt{\left(A^{2}-B^{2}\right) \alpha}-A \sqrt{\alpha} \cos \left(2 \sqrt{\alpha}\left(\xi+\xi_{0}\right)\right)}{A \sin \left(2 \sqrt{\alpha}\left(\xi+\xi_{0}\right)\right)+B}\right)+\alpha\left(\frac{-\sqrt{\left(A^{2}-B^{2}\right) \alpha}-A \sqrt{\alpha} \cos \left(2 \sqrt{\alpha}\left(\xi+\xi_{0}\right)\right)}{A \sin \left(2 \sqrt{\alpha}\left(\xi+\xi_{0}\right)\right)+B}\right)^{-1}\right]^{2}
\end{aligned}
$$$$
\psi_{11}= \pm \sqrt{-n+4 m} e^{i \theta}\left[\left(i \sqrt{\alpha}+\frac{-2 A i \sqrt{\alpha}}{A+\cos \left(2 \sqrt{\alpha}\left(\xi+\xi_{0}\right)\right)-i \sin \left(2 \sqrt{\alpha}\left(\xi+\xi_{0}\right)\right)}\right)+\alpha\left(i \sqrt{\alpha}+\frac{-2 A i \sqrt{\alpha}}{A+\cos \left(2 \sqrt{\alpha}\left(\xi+\xi_{0}\right)\right)-i \sin \left(2 \sqrt{\alpha}\left(\xi+\xi_{0}\right)\right)}\right)^{-1}\right]
$$$$
v_{11}=\frac{-n+4 m}{2 m-1} e^{2 i \theta}\left[\left(i \sqrt{\alpha}+\frac{-2 A i \sqrt{\alpha}}{A+\cos \left(2 \sqrt{\alpha}\left(\xi+\xi_{0}\right)\right)-i \sin \left(2 \sqrt{\alpha}\left(\xi+\xi_{0}\right)\right)}\right)+\alpha\left(i \sqrt{\alpha}+\frac{-2 A i \sqrt{\alpha}}{A+\cos \left(2 \sqrt{\alpha}\left(\xi+\xi_{0}\right)\right)-i \sin \left(2 \sqrt{\alpha}\left(\xi+\xi_{0}\right)\right)}\right)^{-1}\right]^{2}
$$ 


$$
\begin{aligned}
\psi_{12}= \pm \sqrt{-n+4 m} e^{i \theta}\left[\left(-i \sqrt{\alpha}+\frac{2 A i \sqrt{\alpha}}{A+\cos \left(2 \sqrt{\alpha}\left(\xi+\xi_{0}\right)\right)+i \sin \left(2 \sqrt{\alpha}\left(\xi+\xi_{0}\right)\right)}\right)\right. \\
\left.+\alpha\left(-i \sqrt{\alpha}+\frac{2 A i \sqrt{\alpha}}{A+\cos \left(2 \sqrt{\alpha}\left(\xi+\xi_{0}\right)\right)+i \sin \left(2 \sqrt{\alpha}\left(\xi+\xi_{0}\right)\right)}\right)^{-1}\right] \\
v_{12}=\frac{-n+4 m}{2 m-1} e^{2 i \theta}\left[\left(-i \sqrt{b}+\frac{2 A i \sqrt{\alpha}}{A+\cos \left(2 \sqrt{\alpha}\left(\xi+\xi_{0}\right)\right)+i \sin \left(2 \sqrt{\alpha}\left(\xi+\xi_{0}\right)\right)}\right)\right. \\
\left.+\alpha\left(-i \sqrt{\alpha}+\frac{2 A i \sqrt{\alpha}}{A+\cos \left(2 \sqrt{\alpha}\left(\xi+\xi_{0}\right)\right)+i \sin \left(2 \sqrt{\alpha}\left(\xi+\xi_{0}\right)\right)}\right)^{-1}\right]^{2}
\end{aligned}
$$

where $\xi=x-c t, c= \pm 2 \sqrt{-n-4 \alpha}, \alpha>0, A$ and $B$ are two real constants;

$$
\begin{aligned}
& \psi_{13}= \pm \sqrt{-2+4 m} e^{i \theta}\left[\left(\frac{\sqrt{\left(A^{2}-B^{2}\right) \alpha}-A \sqrt{\alpha} \cos \left(2 \sqrt{\alpha}\left(\xi+\xi_{0}\right)\right)}{A \sin \left(2 \sqrt{\alpha}\left(\xi+\xi_{0}\right)\right)+B}\right) \mp \alpha\left(\frac{\sqrt{\left(A^{2}-B^{2}\right) \alpha}-A \sqrt{\alpha} \cos \left(2 \sqrt{\alpha}\left(\xi+\xi_{0}\right)\right)}{A \sin \left(2 \sqrt{\alpha}\left(\xi+\xi_{0}\right)\right)+B}\right)^{-1}\right] \\
& v_{13}=2 e^{2 i \theta}\left[\left(\frac{\sqrt{\left(A^{2}-B^{2}\right) \alpha}-A \sqrt{\alpha} \cos \left(2 \sqrt{\alpha}\left(\xi+\xi_{0}\right)\right)}{A \sin \left(2 \sqrt{\alpha}\left(\xi+\xi_{0}\right)\right)+B}\right) \mp \alpha\left(\frac{\sqrt{\left(A^{2}-B^{2}\right) \alpha}-A \sqrt{b} \cos \left(2 \sqrt{\alpha}\left(\xi+\xi_{0}\right)\right)}{A \sin \left(2 \sqrt{\alpha}\left(\xi+\xi_{0}\right)\right)+B}\right)^{-1}\right]^{2} \\
& \psi_{14}= \pm \sqrt{-2+4 m} e^{i \theta}\left[\left(\frac{-\sqrt{\left(A^{2}-B^{2}\right) \alpha}-A \sqrt{\alpha} \cos \left(2 \sqrt{\alpha}\left(\xi+\xi_{0}\right)\right)}{A \sin \left(2 \sqrt{\alpha}\left(\xi+\xi_{0}\right)\right)+B}\right) \mp \alpha\left(\frac{-\sqrt{\left(A^{2}-B^{2}\right) \alpha}-A \sqrt{\alpha} \cos \left(2 \sqrt{\alpha}\left(\xi+\xi_{0}\right)\right)}{A \sin \left(2 \sqrt{\alpha}\left(\xi+\xi_{0}\right)\right)+B}\right)^{-1}\right] \\
& v_{14}=2 e^{2 i \theta}\left[\left(\frac{-\sqrt{\left(A^{2}-B^{2}\right) \alpha}-A \sqrt{\alpha} \cos \left(2 \sqrt{\alpha}\left(\xi+\xi_{0}\right)\right)}{A \sin \left(2 \sqrt{\alpha}\left(\xi+\xi_{0}\right)\right)+B}\right) \mp \alpha\left(\frac{-\sqrt{\left(A^{2}-B^{2}\right) \alpha}-A \sqrt{\alpha} \cos \left(2 \sqrt{\alpha}\left(\xi+\xi_{0}\right)\right)}{A \sin \left(2 \sqrt{\alpha}\left(\xi+\xi_{0}\right)\right)+B}\right)^{-1}\right]^{2} \\
& \psi_{15}= \pm \sqrt{-2+4 m} e^{i \theta}\left[\left(i \sqrt{\alpha}+\frac{-2 A i \sqrt{\alpha}}{A+\cos \left(2 \sqrt{\alpha}\left(\xi+\xi_{0}\right)\right)-i \sin \left(2 \sqrt{\alpha}\left(\xi+\xi_{0}\right)\right)}\right) \mp \alpha\left(i \sqrt{\alpha}+\frac{-2 A i \sqrt{\alpha}}{A+\cos \left(2 \sqrt{\alpha}\left(\xi+\xi_{0}\right)\right)-i \sin \left(2 \sqrt{\alpha}\left(\xi+\xi_{0}\right)\right)}\right)^{-1}\right] \\
& v_{15}=2 e^{2 i \theta}\left[\left(i \sqrt{\alpha}+\frac{-2 A i \sqrt{\alpha}}{A+\cos \left(2 \sqrt{\alpha}\left(\xi+\xi_{0}\right)\right)-i \sin \left(2 \sqrt{\alpha}\left(\xi+\xi_{0}\right)\right)}\right) \mp \alpha\left(i \sqrt{\alpha}+\frac{-2 A i \sqrt{\alpha}}{A+\cos \left(2 \sqrt{\alpha}\left(\xi+\xi_{0}\right)\right)-i \sin \left(2 \sqrt{\alpha}\left(\xi+\xi_{0}\right)\right)}\right)^{-1}\right]^{2} \\
& \psi_{16}= \pm \sqrt{-2+4 m} e^{i \theta}\left[\left(-i \sqrt{\alpha}+\frac{2 A i \sqrt{\alpha}}{A+\cos \left(2 \sqrt{\alpha}\left(\xi+\xi_{0}\right)\right)+i \sin \left(2 \sqrt{\alpha}\left(\xi+\xi_{0}\right)\right)}\right)\right. \\
& \left.\mp \alpha\left(-i \sqrt{\alpha}+\frac{2 A i \sqrt{\alpha}}{A+\cos \left(2 \sqrt{\alpha}\left(\xi+\xi_{0}\right)\right)+i \sin \left(2 \sqrt{\alpha}\left(\xi+\xi_{0}\right)\right)}\right)^{-1}\right] \\
& v_{16}=2 e^{2 i \theta}\left[\left(-i \sqrt{\alpha}+\frac{2 A i \sqrt{\alpha}}{A+\cos \left(2 \sqrt{\alpha}\left(\xi+\xi_{0}\right)\right)+i \sin \left(2 \sqrt{\alpha}\left(\xi+\xi_{0}\right)\right)}\right) \mp \alpha\left(-i \sqrt{\alpha}+\frac{2 A i \sqrt{\alpha}}{A+\cos \left(2 \sqrt{\alpha}\left(\xi+\xi_{0}\right)\right)+i \sin \left(2 \sqrt{\alpha}\left(\xi+\xi_{0}\right)\right)}\right)^{-1}\right]^{2}
\end{aligned}
$$

where $\xi=x-c t, c= \pm 2 \sqrt{-n+8 \alpha}, \alpha>0, A$ and $B$ are two real constants;

$$
\begin{aligned}
& \psi_{17}= \pm \sqrt{-2+4 m} e^{i \theta}\left(\frac{\sqrt{-\left(A^{2}+B^{2}\right) \alpha}-A \sqrt{-\alpha} \cosh \left(2 \sqrt{-\alpha}\left(\xi+\xi_{0}\right)\right)}{A \sinh \left(2 \sqrt{-\alpha}\left(\xi+\xi_{0}\right)\right)+B}\right) \\
& v_{17}=2 e^{2 i \theta}\left(\frac{\sqrt{-\left(A^{2}+B^{2}\right) \alpha}-A \sqrt{-\alpha} \cosh \left(2 \sqrt{-\alpha}\left(\xi+\xi_{0}\right)\right)}{A \sinh \left(2 \sqrt{-\alpha}\left(\xi+\xi_{0}\right)\right)+B}\right)^{2} \\
& \psi_{18}= \pm \sqrt{-2+4 m} e^{i \theta}\left(\frac{-\sqrt{-\left(A^{2}+B^{2}\right) \alpha}-A \sqrt{-\alpha} \cosh \left(2 \sqrt{-\alpha}\left(\xi+\xi_{0}\right)\right)}{A \sinh \left(2 \sqrt{-\alpha}\left(\xi+\xi_{0}\right)\right)+B}\right) \\
& v_{18}=2 e^{2 i \theta}\left(\frac{-\sqrt{-\left(A^{2}+B^{2}\right) \alpha}-A \sqrt{-\alpha} \cosh \left(2 \sqrt{-\alpha}\left(\xi+\xi_{0}\right)\right)}{A \sinh \left(2 \sqrt{-\alpha}\left(\xi+\xi_{0}\right)\right)+B}\right)^{2}
\end{aligned}
$$




$$
\begin{aligned}
& \psi_{19}= \pm \sqrt{-2+4 m} e^{i \theta}\left(\sqrt{-\alpha}+\frac{-2 A \sqrt{-\alpha}}{A+\cosh \left(2 \sqrt{-\alpha}\left(\xi+\xi_{0}\right)\right)-\sinh \left(2 \sqrt{-\alpha}\left(\xi+\xi_{0}\right)\right)}\right) \\
& v_{19}=2 e^{2 i \theta}\left(\sqrt{-\alpha}+\frac{-2 A \sqrt{-\alpha}}{A+\cosh \left(2 \sqrt{-\alpha}\left(\xi+\xi_{0}\right)\right)-\sinh \left(2 \sqrt{-\alpha}\left(\xi+\xi_{0}\right)\right)}\right)^{2} \\
& \psi_{20}= \pm \sqrt{-2+4 m} e^{i \theta}\left(-\sqrt{-\alpha}+\frac{2 A \sqrt{-\alpha}}{A+\cosh \left(2 \sqrt{-\alpha}\left(\xi+\xi_{0}\right)\right)+\sinh \left(2 \sqrt{-\alpha}\left(\xi+\xi_{0}\right)\right)}\right) \\
& v_{20}=2 e^{2 i \theta}\left(-\sqrt{-\alpha}+\frac{2 A \sqrt{-\alpha}}{A+\cosh \left(2 \sqrt{-\alpha}\left(\xi+\xi_{0}\right)\right)+\sinh \left(2 \sqrt{-\alpha}\left(\xi+\xi_{0}\right)\right)}\right)^{2} \\
& \psi_{21}= \pm \sqrt{-2+4 m} \alpha e^{i \theta}\left(\frac{\sqrt{-\left(A^{2}+B^{2}\right) \alpha}-A \sqrt{-\alpha} \cosh \left(2 \sqrt{-\alpha}\left(\xi+\xi_{0}\right)\right)}{A \sinh \left(2 \sqrt{-\alpha}\left(\xi+\xi_{0}\right)\right)+B}\right)^{-1} \\
& v_{21}=2 \alpha^{2} e^{2 i \theta}\left(\frac{\sqrt{-\left(A^{2}+B^{2}\right) \alpha}-A \sqrt{-\alpha} \cosh \left(2 \sqrt{-\alpha}\left(\xi+\xi_{0}\right)\right)}{A \sinh \left(2 \sqrt{-\alpha}\left(\xi+\xi_{0}\right)\right)+B}\right)^{-2} \\
& \psi_{22}= \pm \sqrt{-2+4 m} \alpha e^{i \theta}\left(\frac{-\sqrt{-\left(A^{2}+B^{2}\right) \alpha}-A \sqrt{-\alpha} \cosh \left(2 \sqrt{-\alpha}\left(\xi+\xi_{0}\right)\right)}{A \sinh \left(2 \sqrt{-\alpha}\left(\xi+\xi_{0}\right)\right)+B}\right)^{-1} \\
& v_{22}=2 \alpha^{2} e^{2 i \theta}\left(\frac{-\sqrt{-\left(A^{2}+B^{2}\right) \alpha}-A \sqrt{-\alpha} \cosh \left(2 \sqrt{-\alpha}\left(\xi+\xi_{0}\right)\right)}{A \sinh \left(2 \sqrt{-\alpha}\left(\xi+\xi_{0}\right)\right)+B}\right)^{-2} \\
& \psi_{23}= \pm \sqrt{-2+4 m} \alpha e^{i \theta}\left(\sqrt{-\alpha}+\frac{-2 A \sqrt{-\alpha}}{A+\cosh \left(2 \sqrt{-\alpha}\left(\xi+\xi_{0}\right)\right)-\sinh \left(2 \sqrt{-\alpha}\left(\xi+\xi_{0}\right)\right)}\right)^{-1} \\
& v_{23}=2 \alpha^{2} e^{2 i \theta}\left(\sqrt{-b}+\frac{-2 A \sqrt{-\alpha}}{A+\cosh \left(2 \sqrt{-\alpha}\left(\xi+\xi_{0}\right)\right)-\sinh \left(2 \sqrt{-\alpha}\left(\xi+\xi_{0}\right)\right)}\right)^{-2} \\
& \psi_{24}= \pm \sqrt{-2+4 m} \alpha e^{i \theta}\left(-\sqrt{-\alpha}+\frac{2 A \sqrt{-\alpha}}{A+\cosh \left(2 \sqrt{-\alpha}\left(\xi+\xi_{0}\right)\right)+\sinh \left(2 \sqrt{-\alpha}\left(\xi+\xi_{0}\right)\right)}\right)^{-1} \\
& v_{24}=2 \alpha^{2} e^{2 i \theta}\left(-\sqrt{-\alpha}+\frac{2 A \sqrt{-\alpha}}{A+\cosh \left(2 \sqrt{-\alpha}\left(\xi+\xi_{0}\right)\right)+\sinh \left(2 \sqrt{-\alpha}\left(\xi+\xi_{0}\right)\right)}\right)^{-2}
\end{aligned}
$$

where $\xi=x-c t, c= \pm 2 \sqrt{-n+2 \alpha}, \alpha<0, A$ and $B$ are two real constants;

$$
\begin{aligned}
& \psi_{25}= \pm \sqrt{-n+4 m} e^{i \theta}\left[\left(\frac{\sqrt{-\left(A^{2}+B^{2}\right) \alpha}-A \sqrt{-\alpha} \cosh \left(2 \sqrt{-\alpha}\left(\xi+\xi_{0}\right)\right)}{A \sinh \left(2 \sqrt{-\alpha}\left(\xi+\xi_{0}\right)\right)+B}\right)+\alpha\left(\frac{\sqrt{-\left(A^{2}+B^{2}\right) \alpha}-A \sqrt{-\alpha} \cosh \left(2 \sqrt{-\alpha}\left(\xi+\xi_{0}\right)\right)}{A \sinh \left(2 \sqrt{-\alpha}\left(\xi+\xi_{0}\right)\right)+B}\right)^{-1}\right] \\
& v_{25}=\frac{-n+4 m}{2 m-1} e^{2 i \theta}\left[\left(\frac{\sqrt{-\left(A^{2}+B^{2}\right) \alpha}-A \sqrt{-\alpha} \cosh \left(2 \sqrt{-\alpha}\left(\xi+\xi_{0}\right)\right)}{A \sinh \left(2 \sqrt{-\alpha}\left(\xi+\xi_{0}\right)\right)+B}\right)+b\left(\frac{\sqrt{-\left(A^{2}+B^{2}\right) \alpha}-A \sqrt{-b} \cosh \left(2 \sqrt{-\alpha}\left(\xi+\xi_{0}\right)\right)}{A \sinh \left(2 \sqrt{-\alpha}\left(\xi+\xi_{0}\right)\right)+B}\right)^{-1}\right]^{2} \\
& \psi_{26}= \pm \sqrt{-n+4 m} e^{i \theta}\left[\left(\frac{-\sqrt{-\left(A^{2}+B^{2}\right) \alpha}-A \sqrt{-\alpha} \cosh \left(2 \sqrt{-\alpha}\left(\xi+\xi_{0}\right)\right)}{A \sinh \left(2 \sqrt{-\alpha}\left(\xi+\xi_{0}\right)\right)+B}\right)+\alpha\left(\frac{-\sqrt{-\left(A^{2}+B^{2}\right) \alpha}-A \sqrt{-\alpha} \cosh \left(2 \sqrt{-\alpha}\left(\xi+\xi_{0}\right)\right)}{A \sinh \left(2 \sqrt{-\alpha}\left(\xi+\xi_{0}\right)\right)+B}\right)^{-1}\right] \\
& v_{26}=\frac{-q+4 p}{2 p-1} e^{2 i \theta}\left[\left(\frac{-\sqrt{-\left(A^{2}+B^{2}\right) \alpha}-A \sqrt{-\alpha} \cosh \left(2 \sqrt{-\alpha}\left(\xi+\xi_{0}\right)\right)}{A \sinh \left(2 \sqrt{-\alpha}\left(\xi+\xi_{0}\right)\right)+B}\right)+\alpha\left(\frac{-\sqrt{-\left(A^{2}+B^{2}\right) \alpha}-A \sqrt{-\alpha} \cosh \left(2 \sqrt{-\alpha}\left(\xi+\xi_{0}\right)\right)}{A \sinh \left(2 \sqrt{-\alpha}\left(\xi+\xi_{0}\right)\right)+B}\right)^{-1}\right]^{2} \\
& \psi_{27}= \pm \sqrt{-n+4 m} e^{i \theta}\left[\left(\sqrt{-\alpha}+\frac{-2 A \sqrt{-\alpha}}{A+\cosh \left(2 \sqrt{-\alpha}\left(\xi+\xi_{0}\right)\right)-\sinh \left(2 \sqrt{-\alpha}\left(\xi+\xi_{0}\right)\right)}\right)\right. \\
& \left.+\alpha\left(\sqrt{-\alpha}+\frac{-2 A \sqrt{-\alpha}}{A+\cosh \left(2 \sqrt{-\alpha}\left(\xi+\xi_{0}\right)\right)-\sinh \left(2 \sqrt{-\alpha}\left(\xi+\xi_{0}\right)\right)}\right)^{-1}\right]
\end{aligned}
$$




$$
\begin{aligned}
& v_{27}=\frac{-n+4 m}{2 m-1} e^{2 i \theta}\left[\left(\sqrt{-\alpha}+\frac{-2 A \sqrt{-\alpha}}{A+\cosh \left(2 \sqrt{-\alpha}\left(\xi+\xi_{0}\right)\right)-\sinh \left(2 \sqrt{-\alpha}\left(\xi+\xi_{0}\right)\right)}\right)\right. \\
& \left.+\alpha\left(\sqrt{-\alpha}+\frac{-2 A \sqrt{-\alpha}}{A+\cosh \left(2 \sqrt{-\alpha}\left(\xi+\xi_{0}\right)\right)-\sinh \left(2 \sqrt{-\alpha}\left(\xi+\xi_{0}\right)\right)}\right)^{-1}\right]^{2} \\
& \psi_{28}=\sqrt{-n+4 m} e^{i \theta}\left[\left(-\sqrt{-\alpha}+\frac{2 A \sqrt{-\alpha}}{A+\cosh \left(2 \sqrt{-\alpha}\left(\xi+\xi_{0}\right)\right)+\sinh \left(2 \sqrt{-\alpha}\left(\xi+\xi_{0}\right)\right)}\right)\right. \\
& \left.+\alpha\left(-\sqrt{-\alpha}+\frac{2 A \sqrt{-\alpha}}{A+\cosh \left(2 \sqrt{-\alpha}\left(\xi+\xi_{0}\right)\right)+\sinh \left(2 \sqrt{-\alpha}\left(\xi+\xi_{0}\right)\right)}\right)^{-1}\right] \\
& v_{28}=\frac{-n+4 m}{2 m-1} e^{2 i \theta}\left[\left(-\sqrt{-\alpha}+\frac{2 A \sqrt{-\alpha}}{A+\cosh \left(2 \sqrt{-\alpha}\left(\xi+\xi_{0}\right)\right)+\sinh \left(2 \sqrt{-\alpha}\left(\xi+\xi_{0}\right)\right)}\right)\right. \\
& \left.+\alpha\left(-\sqrt{-\alpha}+\frac{2 A \sqrt{-\alpha}}{A+\cosh \left(2 \sqrt{-\alpha}\left(\xi+\xi_{0}\right)\right)+\sinh \left(2 \sqrt{-\alpha}\left(\xi+\xi_{0}\right)\right)}\right)^{-1}\right]^{2}
\end{aligned}
$$

where $\xi=x-c t, c= \pm 2 \sqrt{-n-4 \alpha}, \alpha<0, A$ and $B$ are two real constants;

$$
\begin{aligned}
& \psi_{29}= \pm \sqrt{-2+4 m} e^{i \theta}\left[\left(\frac{\sqrt{-\left(A^{2}+B^{2}\right) \alpha}-A \sqrt{-\alpha} \cosh \left(2 \sqrt{-\alpha}\left(\xi+\xi_{0}\right)\right)}{A \sinh \left(2 \sqrt{-\alpha}\left(\xi+\xi_{0}\right)\right)+B}\right) \mp \alpha\left(\frac{\sqrt{-\left(A^{2}+B^{2}\right) \alpha}-A \sqrt{-\alpha} \cosh \left(2 \sqrt{-\alpha}\left(\xi+\xi_{0}\right)\right)}{A \sinh \left(2 \sqrt{-\alpha}\left(\xi+\xi_{0}\right)\right)+B}\right)^{-1}\right] \\
& v_{29}=2 e^{2 i \theta}\left[\left(\frac{\sqrt{-\left(A^{2}+B^{2}\right) \alpha}-A \sqrt{-\alpha} \cosh \left(2 \sqrt{-\alpha}\left(\xi+\xi_{0}\right)\right)}{A \sinh \left(2 \sqrt{-\alpha}\left(\xi+\xi_{0}\right)\right)+B}\right) \mp \alpha\left(\frac{\sqrt{-\left(A^{2}+B^{2}\right) \alpha}-A \sqrt{-\alpha} \cosh \left(2 \sqrt{-\alpha}\left(\xi+\xi_{0}\right)\right)}{A \sinh \left(2 \sqrt{-\alpha}\left(\xi+\xi_{0}\right)\right)+B}\right)^{-1}\right]^{2} \\
& \psi_{30}= \pm \sqrt{-2+4 m} e^{i \theta}\left[\left(\frac{-\sqrt{-\left(A^{2}+B^{2}\right) \alpha}-A \sqrt{-\alpha} \cosh \left(2 \sqrt{-\alpha}\left(\xi+\xi_{0}\right)\right)}{A \sinh \left(2 \sqrt{-\alpha}\left(\xi+\xi_{0}\right)\right)+B}\right)\right. \\
& \left.\mp \alpha\left(\frac{-\sqrt{-\left(A^{2}+B^{2}\right) \alpha}-A \sqrt{-b} \cosh \left(2 \sqrt{-\alpha}\left(\xi+\xi_{0}\right)\right)}{A \sinh \left(2 \sqrt{-\alpha}\left(\xi+\xi_{0}\right)\right)+B}\right)^{-1}\right] \\
& v_{30}=2 e^{2 i \theta}\left[\left(\frac{-\sqrt{-\left(A^{2}+B^{2}\right) \alpha}-A \sqrt{-\alpha} \cosh \left(2 \sqrt{-\alpha}\left(\xi+\xi_{0}\right)\right)}{A \sinh \left(2 \sqrt{-\alpha}\left(\xi+\xi_{0}\right)\right)+B}\right) \mp \alpha\left(\frac{-\sqrt{-\left(A^{2}+B^{2}\right) \alpha}-A \sqrt{-\alpha} \cosh \left(2 \sqrt{-\alpha}\left(\xi+\xi_{0}\right)\right)}{A \sinh \left(2 \sqrt{-\alpha}\left(\xi+\xi_{0}\right)\right)+B}\right)^{-1}\right]^{2} \\
& \psi_{31}= \pm \sqrt{-2+4 m} e^{i \theta}\left[\left(\sqrt{-\alpha}+\frac{-2 A \sqrt{-\alpha}}{A+\cosh \left(2 \sqrt{-\alpha}\left(\xi+\xi_{0}\right)\right)-\sinh \left(2 \sqrt{-\alpha}\left(\xi+\xi_{0}\right)\right)}\right)\right. \\
& \left.\mp \alpha\left(\sqrt{-\alpha}+\frac{-2 A \sqrt{-\alpha}}{A+\cosh \left(2 \sqrt{-\alpha}\left(\xi+\xi_{0}\right)\right)-\sinh \left(2 \sqrt{-\alpha}\left(\xi+\xi_{0}\right)\right)}\right)^{-1}\right] \\
& v_{31}=2 e^{2 i \theta}\left[\left(\sqrt{-\alpha}+\frac{-2 A \sqrt{-\alpha}}{A+\cosh \left(2 \sqrt{-\alpha}\left(\xi+\xi_{0}\right)\right)-\sinh \left(2 \sqrt{-\alpha}\left(\xi+\xi_{0}\right)\right)}\right) \mp \alpha\left(\sqrt{-\alpha}+\frac{-2 A \sqrt{-\alpha}}{A+\cosh \left(2 \sqrt{-\alpha}\left(\xi+\xi_{0}\right)\right)-\sinh \left(2 \sqrt{-\alpha}\left(\xi+\xi_{0}\right)\right)}\right)^{-1}\right]^{2} \\
& \psi_{32}= \pm \sqrt{-2+4 m} e^{i \theta}\left[\left(-\sqrt{-\alpha}+\frac{2 A \sqrt{-\alpha}}{A+\cosh \left(2 \sqrt{-\alpha}\left(\xi+\xi_{0}\right)\right)+\sinh \left(2 \sqrt{-\alpha}\left(\xi+\xi_{0}\right)\right)}\right)\right. \\
& \left.\mp \alpha\left(-\sqrt{-\alpha}+\frac{2 A \sqrt{-\alpha}}{A+\cosh \left(2 \sqrt{-\alpha}\left(\xi+\xi_{0}\right)\right)+\sinh \left(2 \sqrt{-\alpha}\left(\xi+\xi_{0}\right)\right)}\right)^{-1}\right] \\
& v_{32}=2 e^{2 i \theta}\left[\left(-\sqrt{-\alpha}+\frac{2 A \sqrt{-\alpha}}{A+\cosh \left(2 \sqrt{-\alpha}\left(\xi+\xi_{0}\right)\right)+\sinh \left(2 \sqrt{-\alpha}\left(\xi+\xi_{0}\right)\right)}\right) \mp \alpha\left(-\sqrt{-\alpha}+\frac{2 A \sqrt{-\alpha}}{A+\cosh \left(2 \sqrt{-\alpha}\left(\xi+\xi_{0}\right)\right)+\sinh \left(2 \sqrt{-\alpha}\left(\xi+\xi_{0}\right)\right)}\right)^{-1}\right]^{2}
\end{aligned}
$$

where $\xi=x-c t, c= \pm 2 \sqrt{-n+8 \alpha}, \alpha<0, A$ and $B$ are two real constants. 


\section{Graphical Presentations of Some Travelling Wave Solutions}

In this section, we demonstrate the graphical descriptions of some solutions in figures 1-4 with the aid of Mathematica.
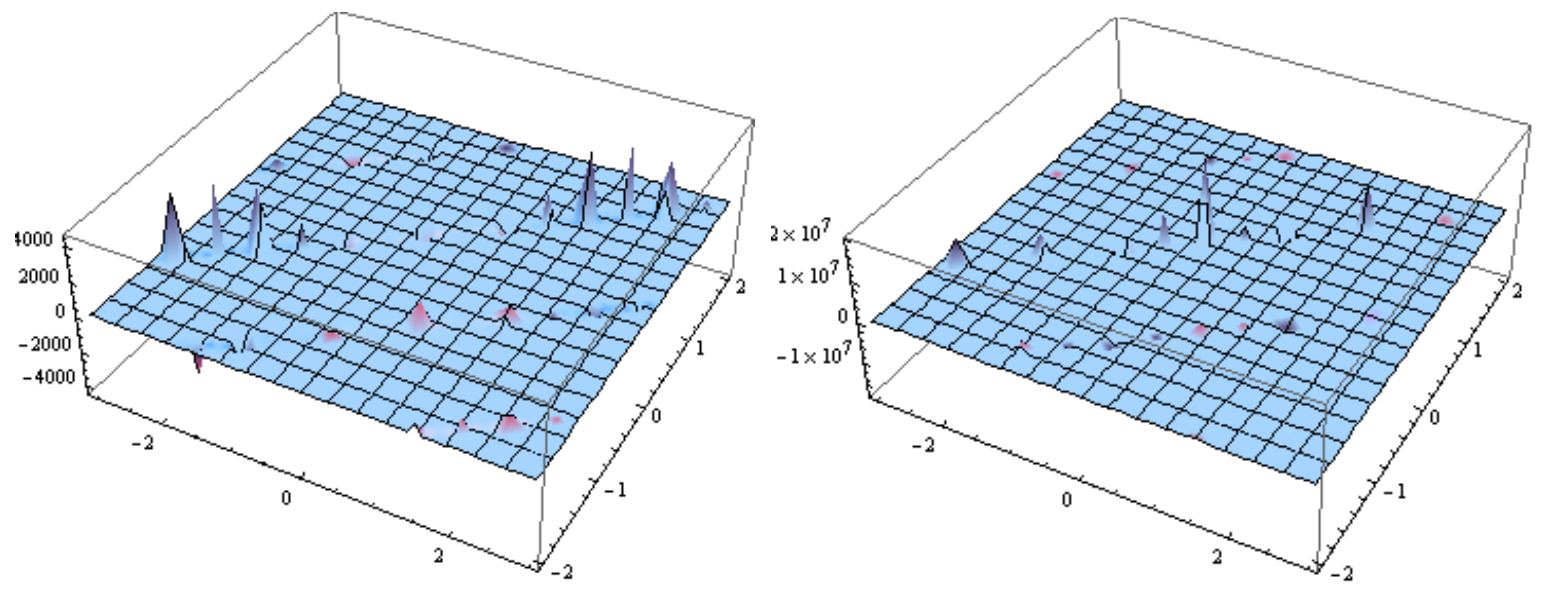

Fig.1. Travelling wave solutions $\operatorname{Re}\left(\boldsymbol{\psi}_{1}\right)$ and $\operatorname{Re}\left(\boldsymbol{v}_{1}\right)$, where $\boldsymbol{\alpha}=\boldsymbol{A}=\boldsymbol{B}=\boldsymbol{m}=\boldsymbol{n}=\mathbf{1}$ and $\boldsymbol{c}=\mathbf{2}$.
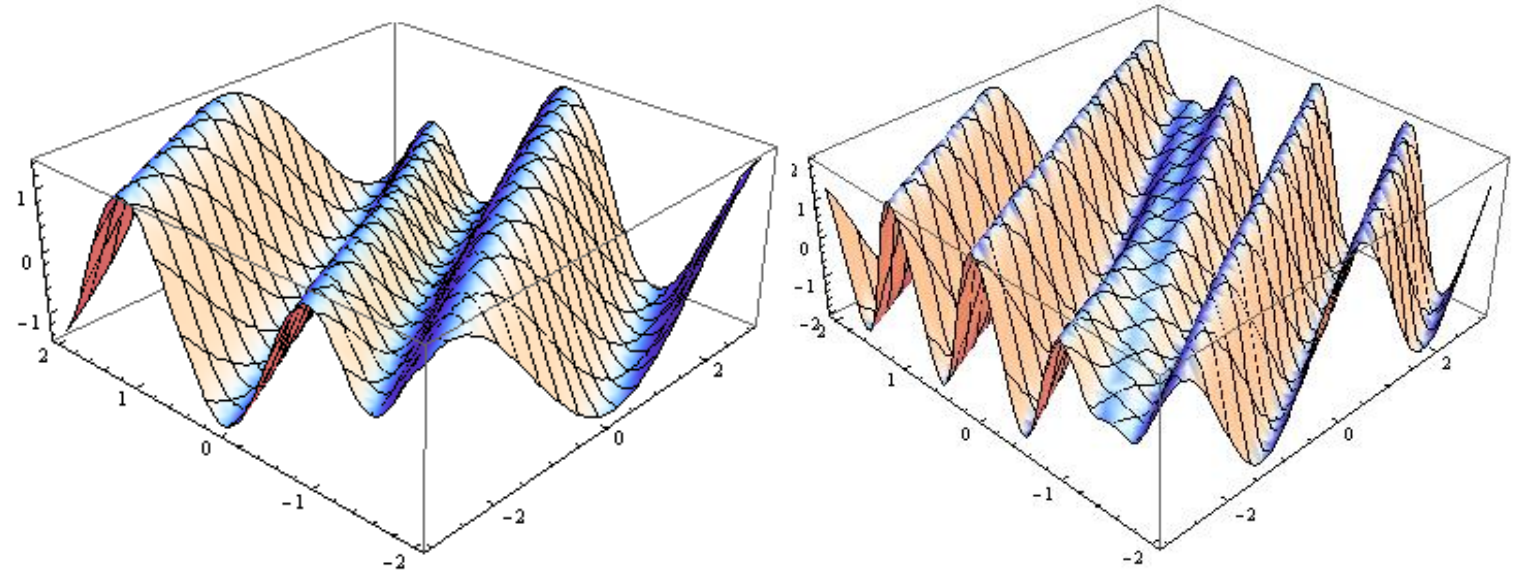

Fig.2. Travelling wave solutions $\operatorname{Re}\left(\boldsymbol{\psi}_{17}\right)$ and $\operatorname{Re}\left(\boldsymbol{v}_{17}\right)$, where $\alpha=-\mathbf{1}, \boldsymbol{A}=\boldsymbol{m}=\mathbf{1}, \boldsymbol{B}=\mathbf{0}, \boldsymbol{n}=-\mathbf{3}$ and $\boldsymbol{c}=\mathbf{2}$.
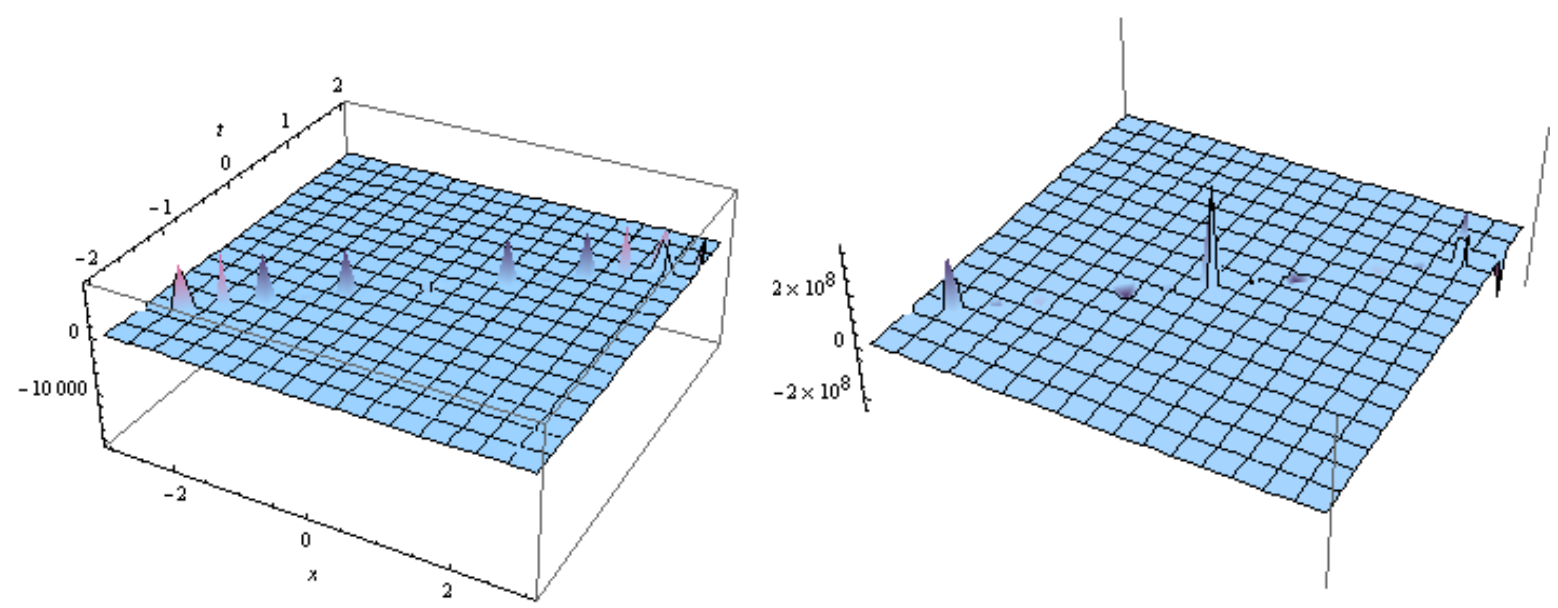

Fig.3. Travelling wave solutions $\operatorname{Re}\left(\boldsymbol{\psi}_{25}\right)$ and $\operatorname{Re}\left(\boldsymbol{v}_{25}\right)$, where $\boldsymbol{\alpha}=-\mathbf{1}, \boldsymbol{A}=\boldsymbol{m}=\mathbf{1}, \boldsymbol{B}=\mathbf{0}, \boldsymbol{n}=\mathbf{3}$ and $\boldsymbol{c}=\mathbf{2}$. 

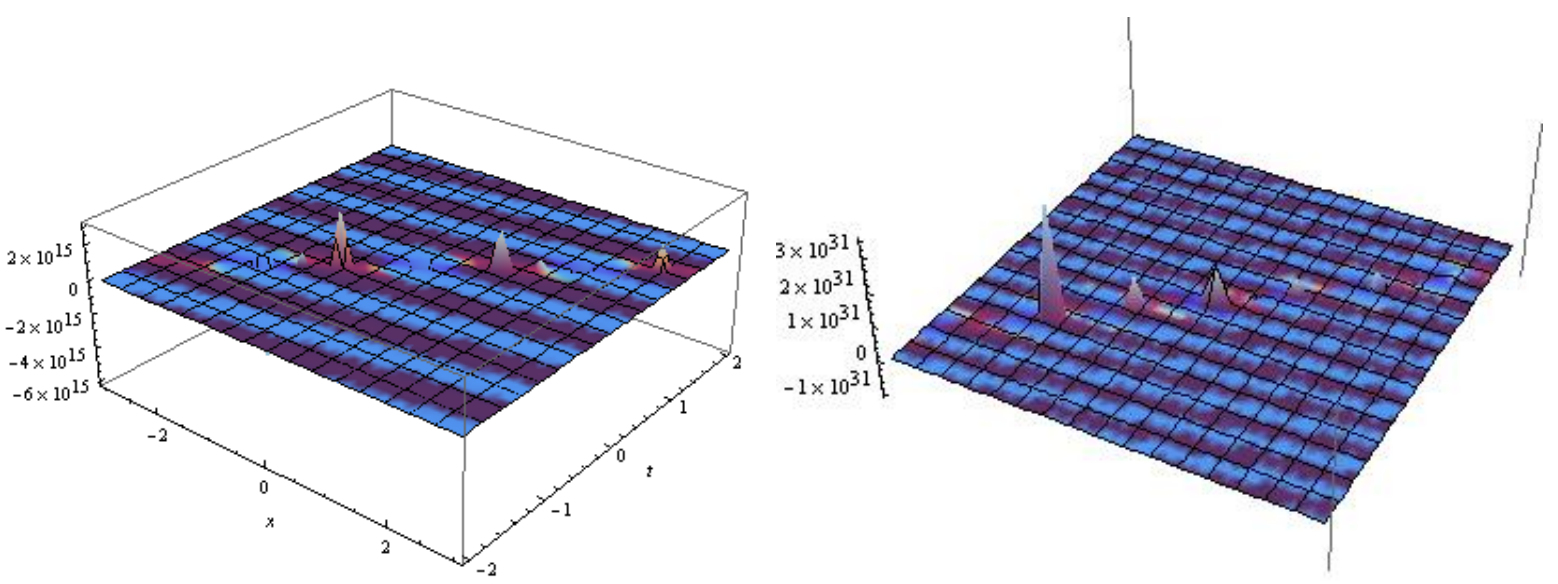

Fig.4. Travelling wave solutions $\operatorname{Re}\left(\boldsymbol{\psi}_{32}\right)$ and $\operatorname{Re}\left(\boldsymbol{v}_{32}\right)$, where $\alpha=-1, A=m=1, n=-9$ and $c=2$.

\section{Conclusion}

The long-short-wave interaction system has been discussed in previous studies and some solutions of this equation system have been obtained by using different methods. In this work, in addition to these solutions, abundant new travelling wave solutions have been obtained via the unified method in straightforward, concise, and elegant manner. Mathematica and Maple facilitates the tedious algebraic calculations.

\section{Acknowledgements}

The author would like to thank the referees for their comments and discussions.

\section{References}

1. Wang, M. L., Wang, Y. M., Zhang, J. L. 2003. The periodic wave solutions for two systems of nonlinear wave equations. Chin. Phys., 12: 1341.

2. Bekir, A., Ünsal, Ö. 2012. Periodic and solitary wave solutions of coupled nonlinear wave equations using the first integral method, Phys. Scr. , 85: 065003.

3. Sirendaoerji. 2017. Constructing infinite number of exact travelling wave solutions of nonlinear evolution equations via an extended tanh-function method, International Journal of Nonlinear Science, 24: 161-168.

4. Huibin, L., Kelin, W. 1990. Exact solutions for some coupled nonlinear equations II, Journal of Physics A: Mathematical and General, 23: 4197-4105.

5. Malfliet, W., Hereman, W. 1996. The tanh method. I: Exact solutions of nonlinear evolution and wave equations, Phys. Scr., 54: 563-568.

6. Fan, E.G. 2000. Extended tanh-function method and its applications to nonlinear equations, Phys Lett. A, 277: 212-218.

7. El-Wakil, S. A. , El-Labany, S. K., Zahran, M. A., Sabry, R. 2005. Modified extended tanhfunction method and its applications to nonlinear equations, Appl. Math. Comput., 161: 403-412.

8. Khuri, S. A. 2004. A complex tanh-function method applied to nonlinear equations of Schrödinger type, Chaos, Solitons and Fractals, 20: 1037-1040. 
9. Wang, M., Li, X., Zhang, J. 2008. The (G'/G)-expansion method and travelling wave solutions of nonlinear evolution equations in mathematical physics, Physics Letters A, 372: 417-423.

10. Gözükızıl, Ö. F., Akçağıl, Ş., Aydemir, T. 2016. Unification of all hyperbolic tangent function methods, Open Phys., 1: 524-541. 